\title{
ARTICLE
}

\section{Transcriptional landscapes at the intersection of neuronal apoptosis and substance P-induced survival: exploring pathways and drug targets}

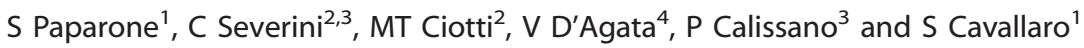

A change in the delicate equilibrium between apoptosis and survival regulates the neurons fate during the development of nervous system and its homeostasis in adulthood. Signaling pathways promoting or protecting from apoptosis are activated by multiple signals, including those elicited by neurotrophic factors, and depend upon specific transcriptional programs. To decipher the rescue program induced by substance P (SP) in cerebellar granule neurons, we analyzed their whole-genome expression profiles after induction of apoptosis and treatment with SP. Transcriptional pathways associated with the survival effect of SP included genes encoding for proteins that may act as pharmacological targets. Inhibition of one of these, the Myc pro-oncogene by treatment with 10058-F4, reverted in a dose-dependent manner the rescue effect of SP. In addition to elucidate the transcriptional mechanisms at the intersection of neuronal apoptosis and survival, our systems biology-based perspective paves the way towards an innovative pharmacology based on targets downstream of neurotrophic factor receptors.

Cell Death Discovery (2016) 2, 16050; doi:10.1038/cddiscovery.2016.50; published online 1 August 2016

\section{INTRODUCTION}

Neuronal apoptosis and survival are orchestrated by intrinsic transcriptional programs regulating. These programs are activated by multiple extracellular and/or intracellular signals, including the absence or presence of neurotrophic factors. Primary cultures of cerebellar granule neurons (CGNs) represent the election model to examine the mechanisms underlying neuronal apoptosis and survival. ${ }^{1}$ In this in vitro paradigm, a rapid apoptotic cell death occurs within $24 \mathrm{~h}$ after removal of serum and lowering of extracellular potassium from 25 to $5 \mathrm{mM}$. Engagement of apoptosis requires transcription and protein synthesis and becomes irreversible after $6 \mathrm{~h}$ from induction. ${ }^{2}$ Before this 'commitment point' CGNs can be rescued by the activation of specific signal-transduction pathways or by the treatment with specific neurotrophic factors, such as insulin-like growth factor-1 (IGF-1), ${ }^{2}$ pituitary adenylyl cyclase-activating polypeptide (PACAP), ${ }^{3,4}$ gastric inhibitory polypeptide $(\mathrm{GIP})^{5}$ and substance $P$ (SP). ${ }^{6}$ Although their effects are mediated by different receptors and intracellular second messengers, their signaling pathways converge into the nucleus and regulate gene expression., ${ }^{3,9}$

The advent of high-throughput technologies is now offering a systems biology-based perspective to analyze the mechanisms underlying neuronal apoptosis and survival. Indeed, the ability of a neuron to promote or evade apoptosis depends on the activity of an integrated network of genes and their encoded proteins, which never work alone but interact with each other in highly structured networks. In recent years, we have begun to explore the systems biology of neuronal apoptosis and survival cross-paths by analyzing whole-genome expression profiles. ${ }^{1,10,11}$ Although our previous studies represent the first glimpse into the transcriptional landscape of neuronal apoptosis and survival, they suggest the existence of a conserved transcriptional program. Indeed, the survival effects of IGF-1 and PACAP share striking similarities and are propagated by common transcriptional cascades. ${ }^{1,7}$ In the present study, we have extended our analysis to SP, a potent antiapoptotic neurotrophic factor, which belongs to the tachykinins neuropeptide family. ${ }^{12}$

\section{RESULTS}

Induction of apoptosis and rescue by SP

As previously demonstrated, apoptosis of CGNs induced by removal of serum and lowering of extracellular potassium from 25 to $5 \mathrm{mM}$ was antagonized by treatment with SP. ${ }^{2}$ The dosedependent rescue effect of SP reached its maximal efficacy (75\%) at $200 \mathrm{nM}$ and was completely reverted by cotreatment with 25 nM SR 140333, a specific antagonist of neurokinin 1 (NK1) receptor (Figures 1a and b). ${ }^{13}$ The presence of NK1 receptors in NeuN-positive CGNs was confirmed by immunocytochemistry analysis (Figure 1c) and supports the direct neuroprotective action of SP on CGNs.

Whole-genome expression changes underlying CGN apoptosis and rescue by SP

We characterized whole-genome expression profiles of CGNs $6 \mathrm{~h}$ after the induction of apoptosis or its rescue by a maximal effective dose of SP (200 nM). Then, we used two complementary approaches to simultaneously investigate changes of individual genes and functional gene groups.

\footnotetext{
${ }^{1}$ Institute of Neurological Sciences, Italian National Research Council, Via Paolo Gaifami, 18, Catania 95125, Italy; ${ }^{2}$ Institute of Cell Biology and Neurobiology, Italian National Research Council, Via del Fosso di Fiorano 64, Roma 00143, Italy; ${ }^{3}$ European Brain Research Institute, Via del Fosso di Fiorano 64, Roma 00143, Italy and ${ }^{4}$ Department of Biomedical and Biotechnological Sciences, Section of Human Anatomy and Histology, University of Catania, Catania 95125, Italy.

Correspondence: S Cavallaro (sebastiano.cavallaro@cnr.it)

Received 4 May 2016; revised 20 May 2016; accepted 25 May 2016; Edited by R Killick
} 


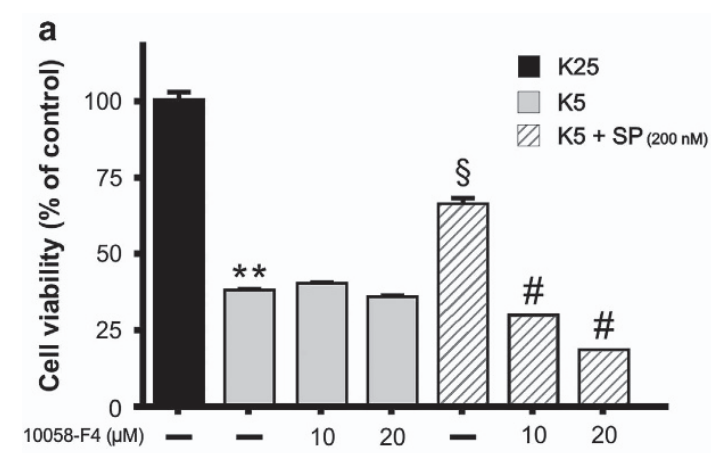

c
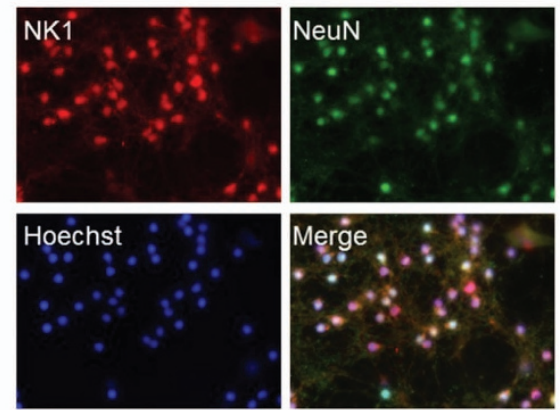

b

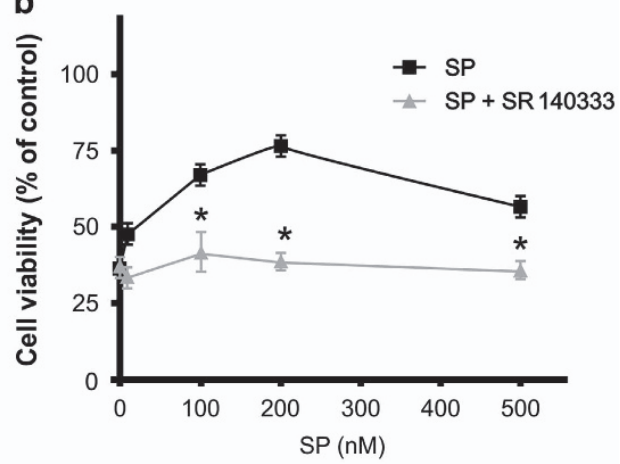

d

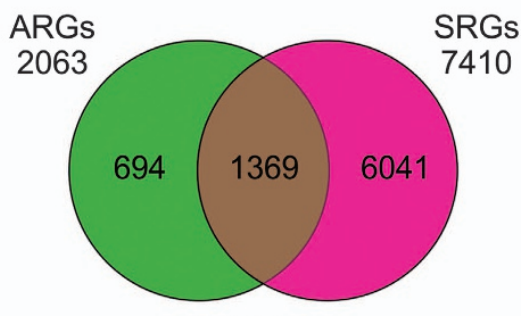

Total 9473

e
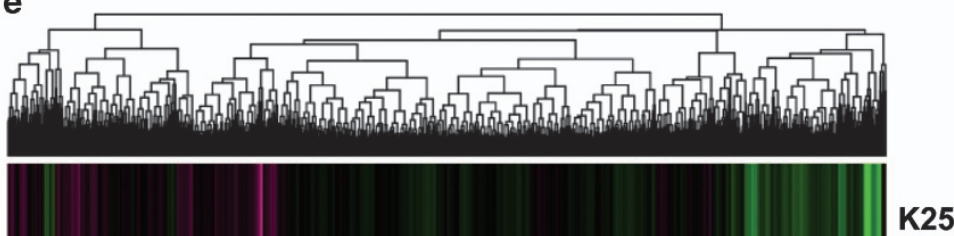

K25

K5

$\mathrm{K} 5+\mathrm{SP}$

Expression

$-2$

0

2

Figure 1. Pharmacological and transcriptional effects of SP following induction of apoptosis in CGNs. (a) Effect of SP and Myc inhibitor on CGNs viability. Primary cultures of CGNs at 6 DIV were switched into serum-free medium containing $5 \mathrm{mM} \mathrm{KCl}$ for an acute induction of apoptotic death. After $48 \mathrm{~h}$, neuronal viability was assessed by counting the number of intact nuclei. Neuroprotective effects of 200 nM SP administration was reverted by cotreatment with the Myc inhibitor. Values for neuronal viability represent the mean \pm S.E.M. of four determinations in two different experiments. Statistically significant differences were calculated by one-way analysis of variance (ANOVA) followed by Bonferroni's test for multiple comparisons $\left({ }^{*} P<0.05,{ }^{* *} P<0.01\right.$ versus $\mathrm{K} 25 ;{ }^{\S} P<0.01$ versus K5; ${ }^{\#} P<0.01$ versus SP treatment). (b) Neuroprotective effects of SP were inhibited by the NK1-selective receptor antagonist SR 140333 (25 nM). (c) Representative immunofluorescence photomicrographs showing CGNs stained with an antibody against NK1 receptor (red) or NeuN to visualize neurons (green). Total nuclei were stained with Hoechst (blue). (d) Genes differentially expressed in control (K25) versus apoptotic (K5) CGNs were defined as ARGs. Genes differentially expressed in apoptotic (K5) CGNs versus those rescued from death by SP treatment (K5+SP) were defined as SRGs. (e) Hierarchical clustering. A hierarchical clustering was used to cluster differentially expressed genes in control (K25), apoptotic (K5) and rescued (K5+SP) CGNs. In this two-dimensional presentation, each row represents a single SRG, whereas the columns represent each of the three different experimental conditions analyzed (K25, K5, K5+SP). As shown in the color bar, the red color indicates upregulation and the green color downregulation.

Differentially expressed genes in apoptotic and rescued CGNs. When gene expression profiles of control (K25) and apoptotic (K5) CGNs were compared, 2063 genes, operationally defined as 'apoptotic related genes' (ARGs), showed significant changes. By comparing gene expression profiles in CGNs $6 \mathrm{~h}$ after the induction of apoptosis (K5) with those of neurons rescued by
SP treatment $(\mathrm{K} 5+\mathrm{SP}), 7410$ genes were found differentially expressed and operationally defined as 'survival-related genes' (SRGs) (Figure 1d). Intersection of ARGs and SRGs comprised 1369 genes (Figure 1d). A comprehensive picture of differentially expressed genes is shown in Figure 1e, where ARGs and SRGs (a total of 9473 genes) are grouped on the basis of similarity in 
their expression patterns with a hierarchical clustering method. The significant impact of apoptosis and survival on the CGN transcriptome can be distinguished by the color matrix.

Deregulated pathways in rescued CGNs by SP. A total of 95 statistically significant pathways were identified in the context of SRGs (Supplementary Data S9). To reduce redundancy of deregulated pathways and simplify their comprehension, the most significant variations implicated in these pathways were summarized in Figures 2-8 and Supplementary Figures 9-15.
Drug targets discovery

Many of statistically altered genes in CGNs rescued by SP encode for drug targets whose modulation might exert antiapoptotic or prosurvival effects (Supplementary Data S10 and S11). Although the pharmacological exploitation of these targets goes beyond the aim of the present study, we functionally analyzed one of these genes, Myc, which is upregulated in rescued versus apoptotic neurons (Figure 2). Treatment of CGNs with different concentrations of the selective Myc inhibitor, 10058-F4, reverted in a dosedependent manner the rescue effect of $200 \mathrm{nM}$ SP (Figure 1a).

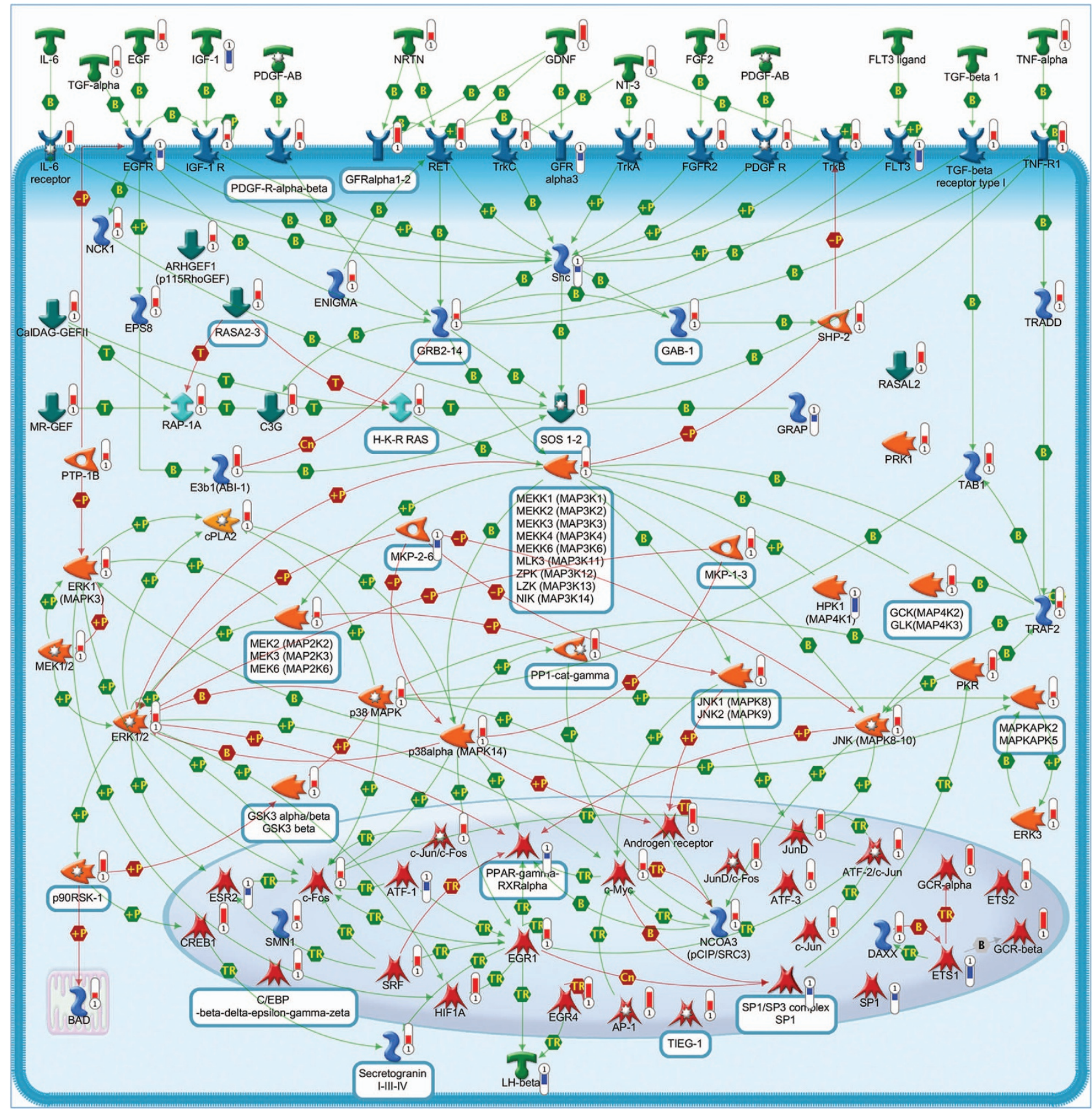

Figure 2. Mitogen-activated protein kinase (MAPK) signaling pathway. The canonical MAPK cascade is activated by a wide variety of stimuli and receptors promoting cell survival and proliferation. This pathway includes a set of adaptors, such as SHC and GRB2, linking the receptor to a guanine nucleotide exchange factor (GEF) such as SOS and C3G, transducing the signal to small GTP-binding proteins (Ras), which in turn activate the core unit of the cascade composed of a MAPKKK (Raf), a MAPKK (MEK1/2 (MAPK/ERK kinase-1/2)) and a MAPK (ERK). An activated ERK dimer, in turn, regulates several targets in the cytosol and translocates to the nucleus where it phosphorylates a variety of transcription factors regulating gene expression. Negative regulators of ERK pathway include MKPs. Pathway objects and links are described separately in the Supplementary Figure 16. 
These data support a direct role of $M y c$ in the neuroprotective activity of SP.

\section{DISCUSSION}

Neuroprotective action of SP is associated with profound transcriptional changes involving different functional categories, each of which will be discussed separately in the following paragraphs.

Signal transduction

CGN rescue by SP involves the different expression of genes encoding for different extracellular mediators and their receptors, as well as numerous downstream proteins of various signaling pathways.

\section{Extracellular mediators}

Neuropeptides: The rescue program induced by SP included the altered expression of several neurotrophic factors, neuropeptides and their receptors notoriously associated with neuroprotective and prosurvival properties (Figures 2, 4 and 6). ${ }^{14}$ Numerous genes encoding for cytokines, chemokines and their corresponding receptors have also showed different expression in rescued CGNs, which are consistent with their role in mediating survival signals. ${ }^{15}$ Moreover, deregulated expression of some interleukins and their receptors is in line with previous studies showing the capacity of these mediators to influence neuronal survival (Figures 2 and 5). ${ }^{16}$

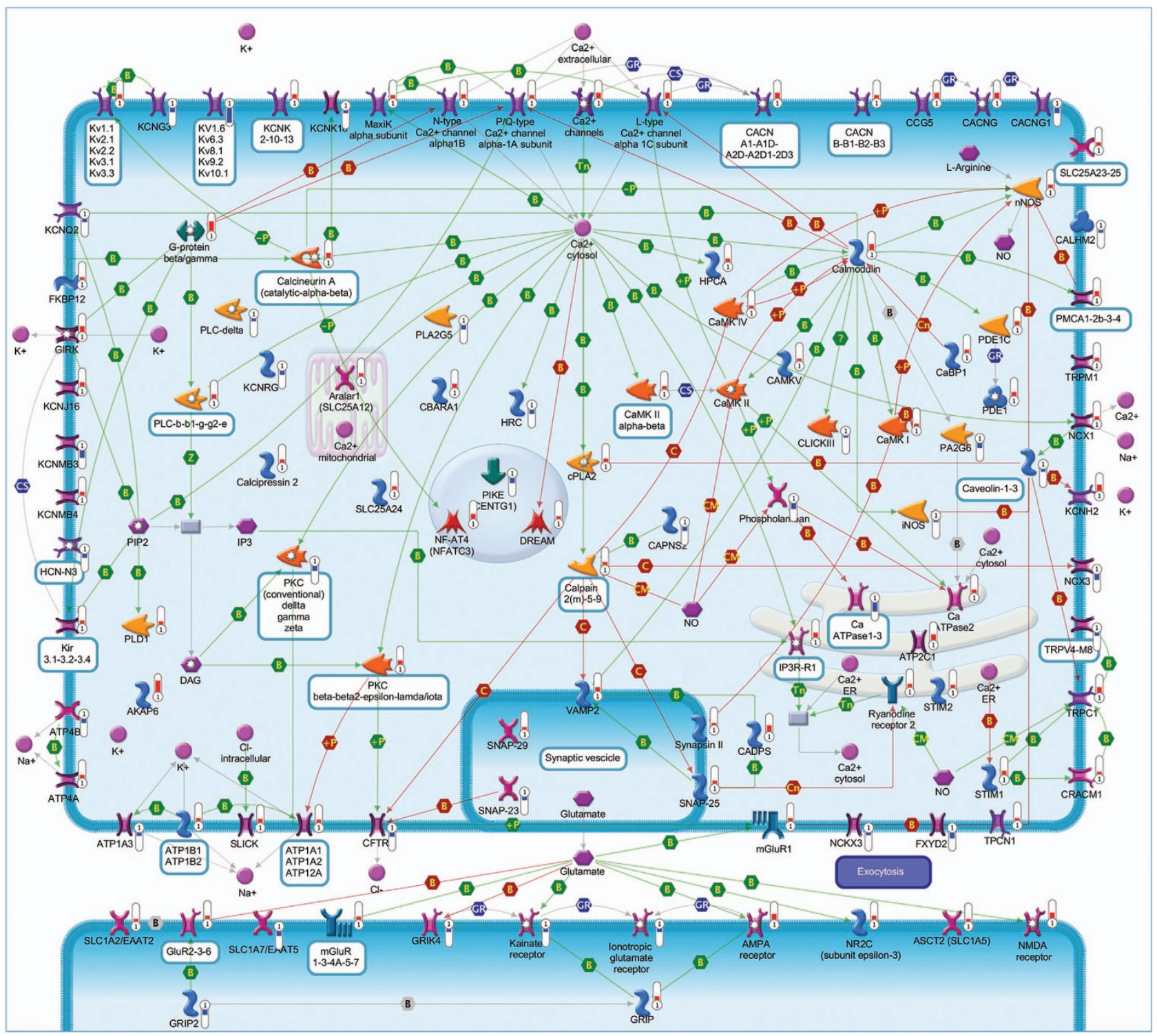

Figure 3. Ion homeostasis. $\mathrm{Ca}^{2+}$ homeostasis is regulated by several transporters. $\mathrm{Ca}^{2+}$ influx is primarily mediated by voltage-gated $\mathrm{Ca}^{2+}$ channels (VGCCs), transient receptor potential channels (TRPs) and ligand-gated channels. $\mathrm{Ca}^{2+}$ efflux is achieved by plasma membrane Ca ${ }^{2+}$ ATPases (PMCAs), sodium calcium exchangers (NCXs) or $\mathrm{Na}^{+} / \mathrm{NCKX}$. Release of $\mathrm{Ca}^{2+}$ from the ER/SR is mediated through IP3 or ryanodine receptors. Store-operated channels are activated by stromal interaction molecule 1 (STIM1) protein that senses the store depletion and triggers the opening of the $\mathrm{Ca}^{2+}$-release-activated $\mathrm{Ca}^{2+}$ channels and calcium release-activated calcium modulators. Store-operated channels entry (SOCE) is also modulated by $\mathrm{K}^{+}$-permeable channels, $\mathrm{KCa}$ and $\mathrm{Kv}$, which are regulated by intracellular $\mathrm{Ca}^{2+}$ and depolarization, respectively. These two channels help sustain SOCE generation by inducing cell hyperpolarization. Additionally, the $\mathrm{Na}^{+}-$permeable channel transient receptor potential cation channel subfamily $\mathrm{M}$ member (TRPM) is gated by intracellular $\mathrm{Ca}^{2+}$ and reduces $\mathrm{Ca}^{2+}$ mobilization. The reuptake of $\mathrm{Ca}^{2+}$ into the ER/SR is primarily mediated by sarcoplasmic/ER Ca ${ }^{2+}$ ATPase. Pathway objects and links are described separately in Supplementary Figure 16. 
Neurotransmitter receptors: The neuroprotective effects of SP were associated with the differential expression of genes encoding for both inhibitory and excitatory neurotransmitter receptors: GABA-A, GABA-B, NMDA, AMPA, kainate and metabotropic glutamate, which are known to profoundly affect neuronal fate (Figure 3 and Supplementary Figure 9). ${ }^{17,18}$ In addition to these, we observed the differential expression of adrenergic, dopaminergic, serotonergic and adenosine receptors, which may further contribute to the fine regulation of neuronal survival (Figure 6). ${ }^{19-21}$

Intracellular signaling pathways. In accordance with the crucial role of the MAPK pathway in mediating the survival effects of IGF-1 and PACAP, ${ }^{1}$ several members of this pathway were found overexpressed following SP treatment (Figure 2). A prominent neuroprotective role in CGNs is also played by the PKA pathway. Consistently, we observed the upregulation of different subunits of PKA in rescued CGNs, including anchoring and interacting proteins, together with different adenylate cyclases and different upstream receptors of this pathway (Figure 6). Such changes are in line with previous findings showing the ability of these proteins to promote survival in serum-deprived neurons. ${ }^{22}$ Upregulation of various components of the PI3K/AKT pathway also confirms the need to maintain this pathway activated to prevent neuronal apoptosis (Figure 4). ${ }^{23}$ The increased expression of different components of the STAT and WNT pathways provides further evidence of their involvement in promoting neuronal survival and inhibiting the death process (Figures 4 and 5). ${ }^{23,24}$ In agreement with the ability of different PKC isoforms to regulate apoptosis and survival, ${ }^{25}$ SP exerted a double activity on the PKC pathway (Figure 3). ${ }^{26}$

Ion homeostasis. Conforming with the ability of IGF-1 and PACAP to induce transcripts encoding for proteins controlling calcium

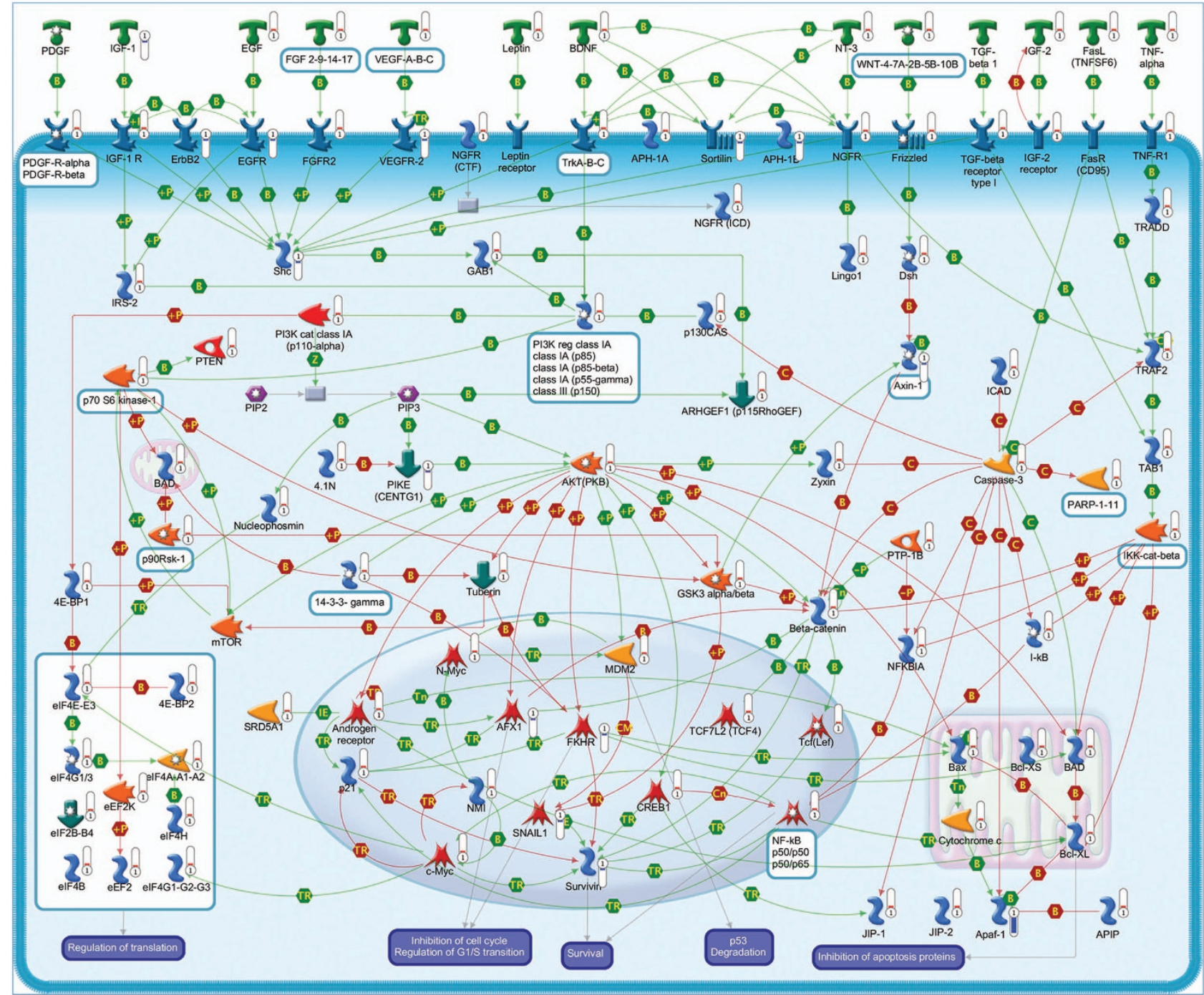

Figure 4. PI3K-AKT (phosphatidylinositol-4,5-bisphosphate 3-kinase-AKT) signaling pathway. The PI3K-PKB/AKT pathway is controlled by a multistep process. Activated receptors directly stimulate class $1 \mathrm{~A}$ PI3Ks bound via their regulatory subunit or adapter molecules such as the insulin receptor substrate (IRS) proteins. This triggers activation of PI3K and conversion of PIP2 (phosphatidylinositol-4,5-bisphosphate) into PIP3 (phosphatidylinositol3,4,5-trisphosphate). PKB/AKT binds to PIP3 at the plasma membrane, allowing PDK1 to activate PKB/AKT. Physiological roles of AKT include activation of protein synthesis, inhibition of apoptosis, cell cycle progression and transcription factors regulation. These are exerted by phosphorylation of a variety of downstream substrates, including caspase-3, BAX, BAD, Bcl-XL, FKHR, IKK, NF- $K B$, GSK3, AR, MDM2, CREB, p21, p70S6K1, JIP1 scaffold protein and a series of initiation factors. Dephosphorylation by protein phosphatase-2A (PP2A) and the conversion of PIP3 to PIP2 by PTEN antagonize AKT signaling. Pathway objects and links are described separately in Supplementary Figure 16. 


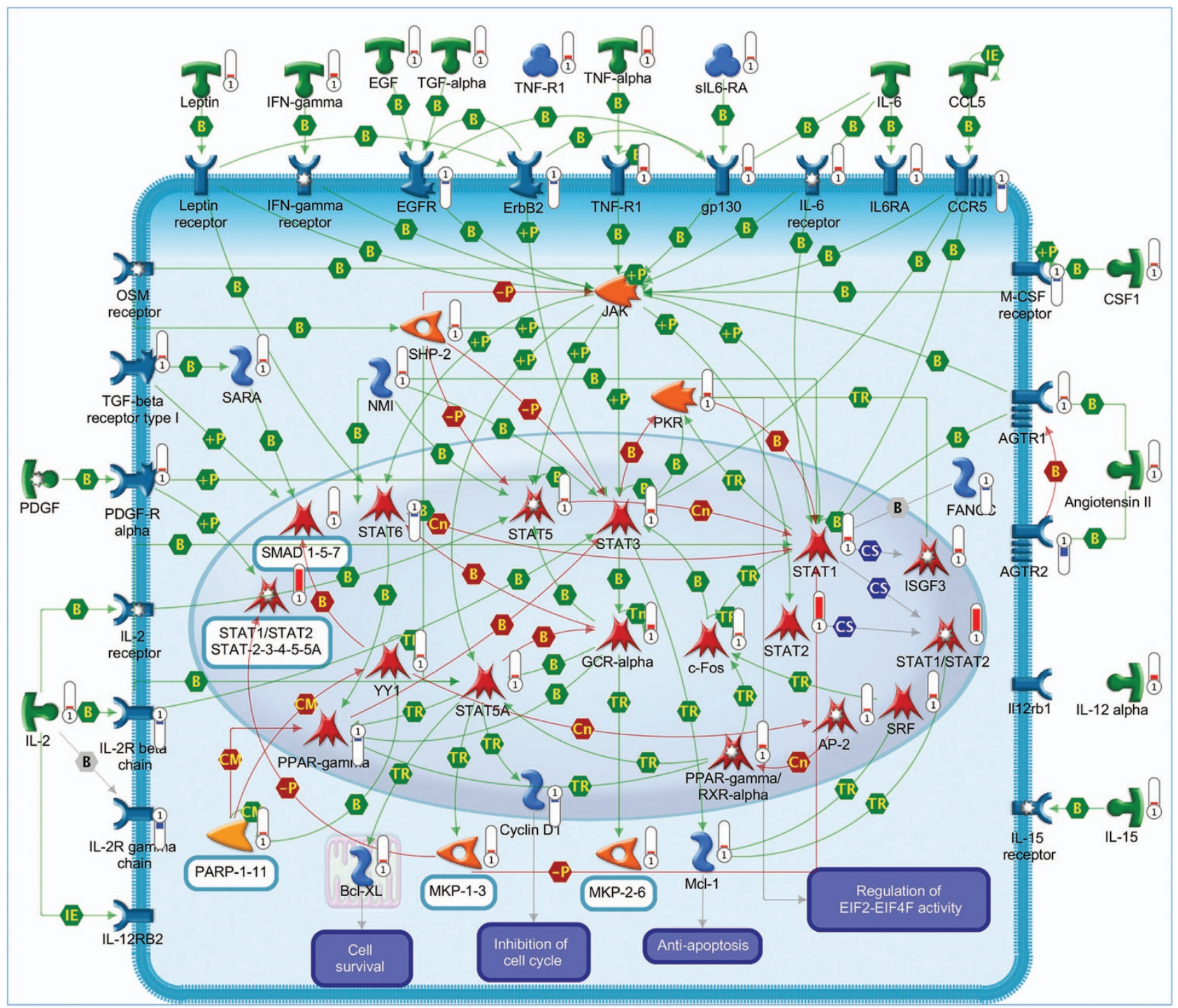

Figure 5. JAK-STAT (Janus kinase/signal transducers and activators of transcription) pathway. The JAK-STAT system consists of three main components: (1) a membrane receptor, (2) JAK and (3) the signal transducer and activator of transcription (STAT). It is activated by different signals, including interferon, interleukin, growth factors and other chemical messengers that activate the kinase function of JAK, which autophosphorylates itself. The STAT proteins then bind the phosphorylated receptor, where they are phosphorylated by JAK. The phosphorylated STAT proteins bind to another phosphorylated STAT protein (dimerizes) and translocate into the nucleus where promote transcription of genes responsive to STAT. The pathway is negatively regulated at multiple levels. Pathway objects and links are described separately in Supplementary Figure 16.

homeostasis during apoptosis rescue, ${ }^{1}$ SP elicited the overexpression of numerous genes regulating the intracellular concentration of this ion. Upregulation of these genes, together with those encoding for proteins that mediate $\mathrm{Ca}^{2+}$ responses or $\mathrm{Ca}^{2+}$ dependent transcription, support their role in preventing cell death and neurodegeneration. ${ }^{27}$ Deregulated genes following SP treatment also included those encoding for different potassium channels and other transporters known to promote prosurvival effects (Figure 3). ${ }^{28}$

Cell adhesion, cytoskeleton remodeling and endosomal/lysosomal pathway

Cell adhesion. CGNs rescued by SP showed the altered expression of genes encoding for various components of focal adhesions, including 14 extracellular matrix proteins, 12 subunits of integrins and their receptors and 9 intracellular proteins. These changes are consistent with the known role of these proteins to provide a suitable substrate for neuronal survival. ${ }^{29}$ Similarly, the upregulation of several components of adherens junctions highlights the importance of this adhesion system in promoting neuronal survival and limiting neurodegeneration. ${ }^{30}$ Overexpression of genes encoding numerous components of tight and gap junctions, as well as those encoding for two desmosomal proteins, supports a possible involvement of these adhesion systems in neuronal activity and survival. ${ }^{31}$ We also observed the differential expression of some matrix metalloproteinases, together with the metallopeptidase inhibitor Timp1, which are associated with neuroprotection (Figure 7 ). ${ }^{32}$

Cytoskeleton remodeling. Changes in transcript levels of various structural and regulatory components of myosin filaments, cytoskeleton, actin-binding proteins, such as actin cytoskeletal and tubulin, support the general role of cytoskeleton in neuronal apoptosis. $^{33}$ In addition, the altered expression of different dyneins, dynactins and kinesins supports the need of an efficient 


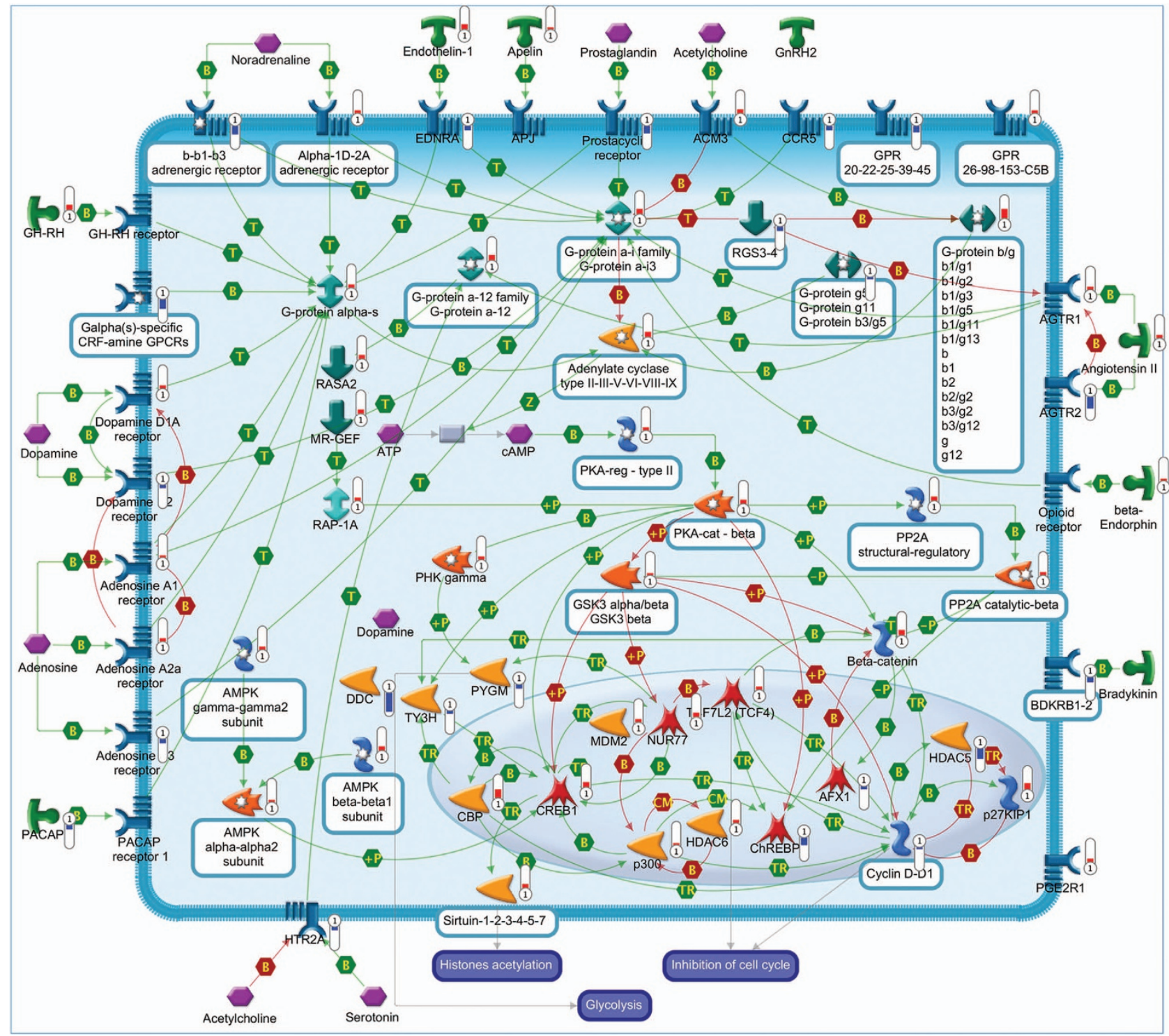

Figure 6. Protein kinase A (PKA) signaling pathway. The majority of G-protein coupled receptors (GPCR) are associated with activation of distinctive adenylate cyclases (ACs) to regulate intracellular CAMP levels. When active, AC produces the second messenger CAMP in response to a wide range of signal-transduction pathways. The main target of cAMP is PKA. Some of the major substrates of PKA include GSK3. PKA phosphorylates and inactivates GSK3, preventing neurodegeneration. PKA also phosphorylates the transcription factor CREB, which in turn allows the recruitment of the coactivator CREB-binding protein (CBP). Thus, PKA is important for an increasing number of physiological processes, such as regulation of the cell cycle that involves chromatin condensation and decondensation. Apart from PKA, other direct targets of cAMP include PDE, p70S6K/RPS6KB1 and PLA2. Pathway objects and links are described separately in Supplementary Figure 16.

retrograde and anterograde axonal transport for ensuring neuronal survival. ${ }^{34}$ Differential expression of various ephrins points out the role of axonal guidance and neuronal outgrowth in neuronal vulnerability (Figure 8 ). ${ }^{35}$

Endosomal/lysosomal pathway. Changes in this pathway profoundly influence cell survival by regulating the expression of specific proteins on the cell surface through their internalization and lysosomal degradation. Consistent with this, CGNs rescued by SP displayed the altered expression of various endosomallysosomal markers, such as the endosomal GTPases Rab. This is in line with previous data showing the ability of this system to mediate neuroprotective effects. ${ }^{36}$ In addition, we identified the differential expression of four vesicle-associated membrane proteins and three synaptosomal-associated proteins, which are correlated with cell viability (Supplementary Figure 9). ${ }^{37}$

\section{Metabolism}

Energy metabolism. Consistent with the rapid decrease in energy metabolism occurring in apoptotic neurons, ${ }^{38}$ CGNs rescued by SP showed the coordinated overexpression of transcripts for 3 glucose transporters, 11 glycolysis enzymes, 13 Krebs cycle enzymes and 29 different subunits of the oxidative phosphorylation complexes. These changes are consistent with the ability of energy to favor neuronal survival. ${ }^{39}$ SP neuroprotective action was also associated with the upregulation of genes encoding for 12 enzymes involved in $\beta$-oxidation that further reflects the need of a greater energy demand for the implementation of survival and apoptotic programs. ${ }^{40}$ In response to the treatment with SP, we also detected the overexpression of the cellular energy status sensor Prkaa2 whose inhibition sensitize neuronal cells against apoptotic death (Supplementary Figure 10). ${ }^{41}$ 


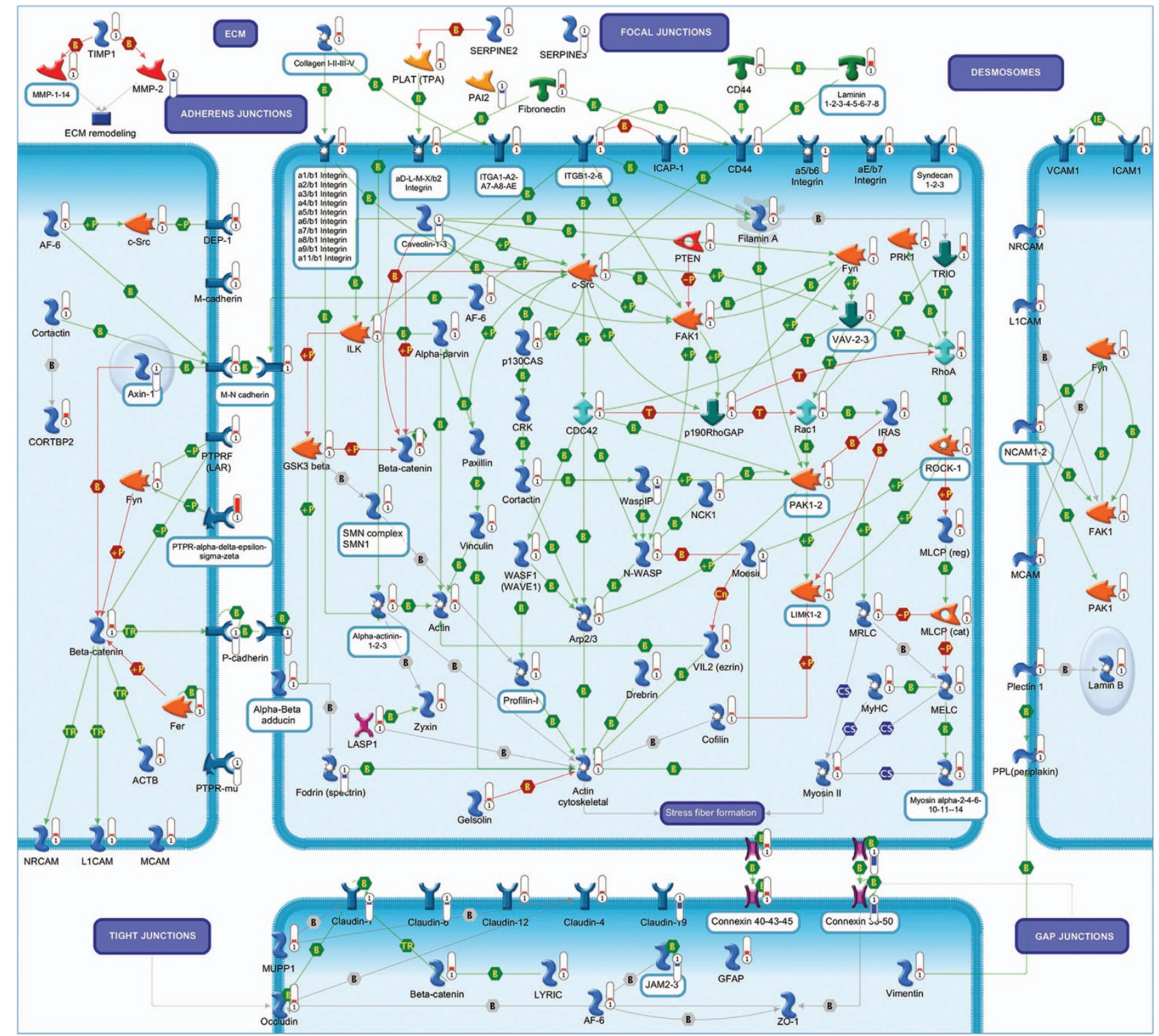

Figure 7. Cell adhesion. Focal adhesions are complexes through which signals are transmitted to extracellular matrix (ECM) and interacting cells. Connection between focal adhesions and ECM is realized through subcellular structures, including integrins that bind to extracellular proteins. The intracellular domain of integrin binds to the cytoskeleton via adapter proteins. Other intracellular proteins associate with this complex. Adherens junctions are subapical structures that function as mediators of cell-cell adhesion. They have a crucial role as sensors of extracellular stimuli. Cadherins are principally responsible for cell-cell adhesion. The extracellular domain of cadherin forms complexes with the extracellular domains of cadherin on neighboring cells. The cytoplasmic domain of cadherin associates with catenins, which provide anchorage to the actin cytoskeleton. Tight junctions are areas of two cells forming an impermeable barrier. They are composed of sealing strands, each of which is formed from a row of transmembrane proteins embedded in both plasma membranes, with extracellular domains joining one another directly. Although different proteins are present, the major are claudins and occludins. These associate with different membrane proteins that anchor the strands to the actin cytoskeleton. Gap junctions are transmembrane channels that connect the cytoplasm of two cells, allowing various molecules to directly pass. Each single channel comprises two hemichannels composed by connexin proteins. The cytoplasmic domain of connexins binds to ZO-1, allowing the association between the gap and tight junctions. Desmosomes are structures specialized for cell-to-cell adhesion, arranged on the sides of plasma membrane and linking surface proteins to intracellular keratin filaments. The inner dense plaque proteins are attached to intermediate filaments. Pathway objects and links are described separately in Supplementary Figure 16.

Transcriptional, translational and epigenetic regulation

Transcriptional regulation. In agreement with the rescue effect of IGF-1 and PACAP, ${ }^{1}$ SP treatment upregulates Jun, Fos and Srf genes, together with other prosurvival transcription factors (Figures 2, 4-6 and Supplementary Figure 11). ${ }^{42}$

Translation regulation. Differential expression of a remarkable number of aminoacyl-tRNA synthetases and other factors implicated in translation in rescue CGNs is compatible with the need for a correct protein synthesis during neuronal survival (Figure 4 and Supplementary Figure 11). ${ }^{43}$

Epigenetic mechanism. The increased expression of the histone acetyltransferases (HATs) and the loss of function of several histone deacetylases (HDACs) have a crucial role in neuronal survival. Upregulation of numerous HATs and other genes 


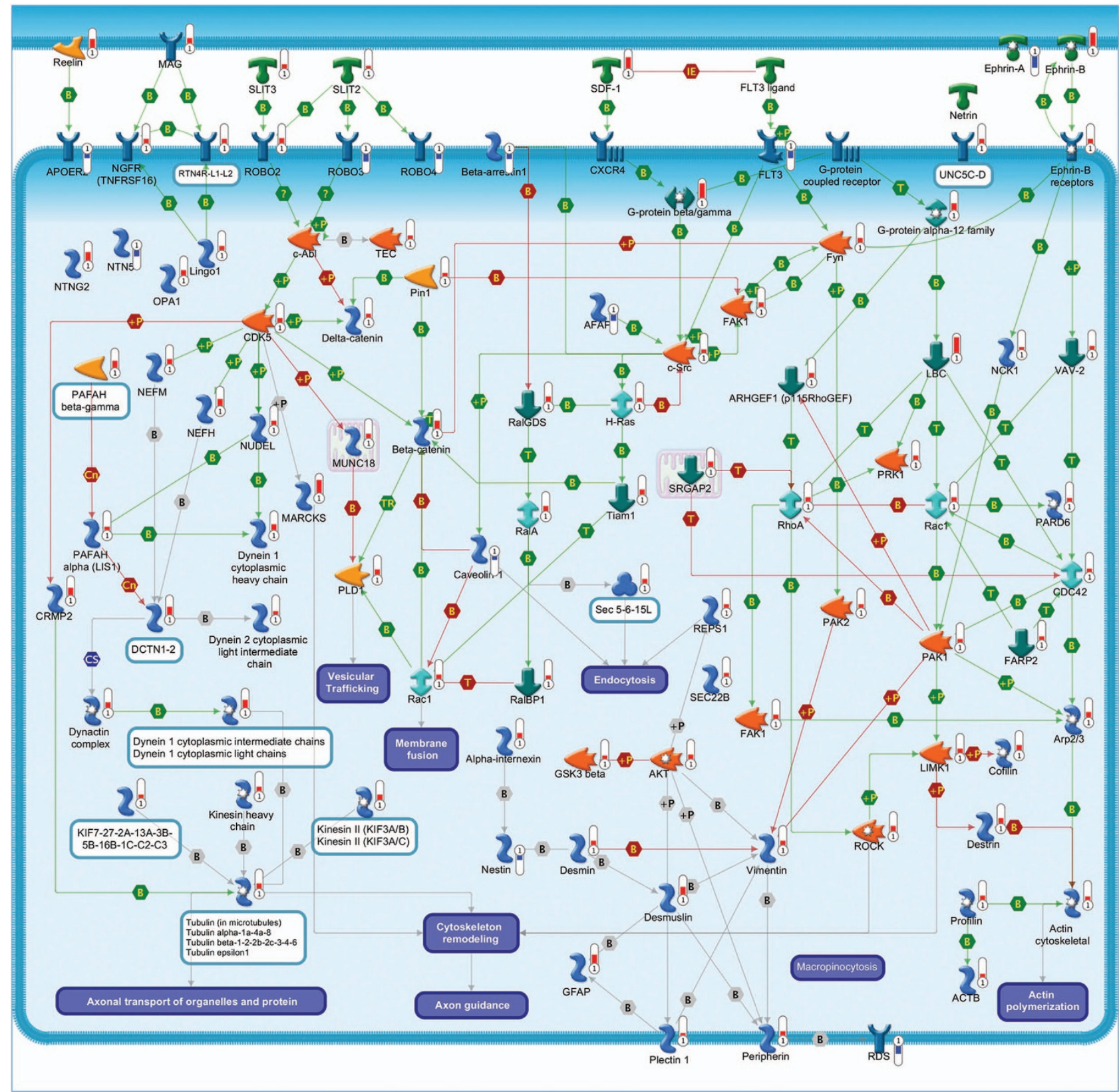

Figure 8. Cytoskeleton remodeling and axonal transport. The cytoskeleton consists of three distinct, yet interconnected filament systems: microfilaments (actin filaments), intermediate filaments (IFs) and microtubules. Microfilaments are the thinnest filaments of the cytoskeleton and are composed of linear polymers of actin subunits. They act as tracks for the movement of myosin molecules and are controlled by the Rho family of small GTP-binding proteins such as Rho, Rac and Cdc42. Intermediate filaments (vimentin, desmin, desmuslin, glial fibrillary acidic protein, peripherin, nestin, $a$-internexin) organize the internal tridimensional structure of the cell, anchoring organelles and serving as structural components of the nuclear lamina. Neurofilaments are the principal intermediate filament type expressed by neurons and are formed by coassembly of neurofilament light polypeptide (NEFL), neurofilament medium polypeptide (NEFM) and heavy neurofilament subunit (NEFH) subunits. Peripherin is another IF protein. Neurofilaments are important protein cargoes for actin-associated motors, such as myosin and microtubule-associated motor, such as kinesin. Microtubules are polymers of $\alpha$ - and $\beta$-tubulin and, in association with other proteins such as dynein and dynactin, are important for axonal transport. Ephrins and their receptor tyrosine kinases play a pivotal role in axon guidance and synaptogenesis. Ephrin-A activation leads to the actin cytoskeleton reorganization and endocytosis. Ephrin-A signaling also promotes neurite outgrowth. Ephrin receptors also maintain feedback mechanisms that reverse signaling through their ephrin ligands. Clustering of ephrin-A molecules with ephrin-A receptors increases cellular adhesion. Conversely, activation of ephrin-B1 leads to the disassembly of focal adhesions. Pathway objects and links are described separately in Supplementary Figure 16.

regulating a series of epigenetic changes supports their neuroprotective action. ${ }^{44}$ Increased expression of DNA methylase, histone methylase and distone demethylase transcripts reinforce the hypothesis of a possible deregulation of DNA and histone methylation during neuronal death. ${ }^{45}$ In addition, upregulation of some histone variants and histone kinase Tlk2 might further contribute to the gene expression regulation in rescued CGNs (Supplementary Figure 12). 
Cell cycle

The resume of quiescent cell cycle caused by the functional alteration of a series of cell cycle regulators is a key event in neuronal apoptosis. ${ }^{46}$ Changes in expression of these regulators observed in rescued CGNs confirm their role in promoting cell survival and contributing to the arrest of cell cycle. At the same time, downregulation of $C d c 25 b$ and Wee 1 is consistent with their known role in promoting S-phase progression and triggering apoptosis. ${ }^{47}$ Similarly, increased expression of the CDK inhibitors supports their potential role in protecting CGNs during $\mathrm{KCl}$ withdrawal-induced apoptosis by inducing cell cycle arrest (Supplementary Figure 13). ${ }^{48}$

\section{Defense mechanisms}

Although neurons are the most vulnerable cells to ROS increase, they possess a series of neuroprotective mechanisms based on antioxidant systems. Upregulation of genes encoding antioxidant or glutathione metabolism proteins supports the hypothesis of an increased oxidative stress and mitochondrial dysfunction in vulnerable neuronal populations and their role in protecting CGNs from oxidative stress-induced apoptosis. ${ }^{49}$ Increased expression of proteins involved in DNA repairing might further contribute to the protection of CGNs against cell death induced by oxidative stress (Supplementary Figure 14).

To counteract multiple signals triggering apoptosis, cells devise a large number of prosurvival strategies involving activation of different Hsps and antiapoptotic factors. CGNs rescued by SP showed the upregulation of numerous Hsps underlining the importance of these proteins in the survival mechanism induced by trophic factors (Figure 4 and Supplementary Figure 14). ${ }^{50}$

Ubiquitin-proteasome system. Defects in the ubiquitin-proteasome system profoundly influence the neuronal viability. ${ }^{51}$ In line with this, rescued CGNs exhibited the coordinated upregulation of different subunits of proteasome $26 \mathrm{~S}$ complex and components of ubiquitin ligases complexes, including adaptor, scaffold and F-box proteins (Supplementary Figure 15).

\section{CONCLUSION}

This work represents a glimpse of the complex transcriptional programs underlying apoptosis and its rescue by SP. In addition to elucidating the common mechanism by which neurotrophic factors mediate CGN survival, many of the genes associated with SP survival effect encode for drug targets whose modulation might exert antiapoptotic or prosurvival effects. Although the functional exploitation of these targets goes beyond the aim of the present study, we pharmacologically inhibited one of these targets, Myc, showing its direct involvement in the neuroprotective activity of SP. The systems biology-based perspective described here, therefore, may pave the way towards an innovative pharmacology based on targets that are downstream of neurotrophic factor receptors.

\section{MATERIALS AND METHODS}

Materials

All the substances were obtained from Sigma-Aldrich (Milano, Italy), unless otherwise specified.

\section{Experimental procedure}

Neuronal cultures. Experimental animal procedures were reviewed and approved by the Institution Committee and carried out in accordance with the European Communities Council Directive of 24 November 1986 (86/609/EEC). Primary cultures of CGNs were obtained from dissociated cerebella of 8-day-old (P8) Wistar rats (Charles River) and prepared as described previously. ${ }^{1}$ After 6 days in vitro (DIV), extracellular $\mathrm{KCl}$ was shifted from 25 to $5 \mathrm{mM}$ for neuronal apoptosis induction. After two washes with serum-free basal medium Eagle containing $5 \mathrm{mM} \mathrm{KCl}$, neurons were incubated with the same medium (K5) or treated with different concentrations of SP $(10,50,100,200$ and $500 \mathrm{nM})$ or the Myc inhibitor, $10058-\mathrm{F} 4(10,20 \mu \mathrm{M})$. Control neurons were grown in serum-free medium supplemented with $25 \mathrm{mM} \mathrm{KCl}(\mathrm{K} 25)$. Neuronal viability was assessed $48 \mathrm{~h}$ later by counting the number of intact nuclei according to the method described previously. ${ }^{13}$

Immunocytochemistry. Experiments were carried out as specified previously. ${ }^{52}$ Briefly, CGNs were fixed with $4 \%$ paraformaldehyde for $15 \mathrm{~min}$ and then incubated in $1 \%$ bovine serum albumin, 10\% normal goat serum, $0.3 \mathrm{M}$ glycine in $0.1 \%$ phosphate-buffered saline (PBS)-Tween for $1 \mathrm{~h}$ to permeabilize them and block nonspecific protein-protein interactions. Cells were then incubated with the primary polyclonal antibody raised against NK1 receptor (1:400 in PBS; Abcam, Cambridge, UK) or NeuN (1 : 200; Sigma-Aldrich) overnight at $+4{ }^{\circ} \mathrm{C}$, and then washed in PBS and incubated with a goat anti-rabbit rhodamine-conjugated secondary antibody (1:1000; Sigma-Aldrich) for $30 \mathrm{~min}$ at room temperature. Cells were also stained with Hoechst $33258(250 \mathrm{ng} / \mathrm{ml})$ for $5 \mathrm{~min}$ at room temperature to label neuronal nuclei. Neurons were then visualized by fluorescent microscopy. ${ }^{52}$

Microarray experiments and data analysis. Whole-genome expression analysis was performed in serum-deprived cells $6 \mathrm{~h}$ after the induction of neuronal apoptosis and treatments with the maximal effective dose of SP $(200 \mathrm{nM})$. RNA extraction, integrity, labeling and hybridization were performed following the protocols outlined by the manufacturers as described previously. ${ }^{1}$ Microarrays were scanned at $5-\mu \mathrm{m}$ resolution using a GenePix Personal 4100A Microarray Scanner and the GenePix Pro 6.0 Acquisition and Data-Extraction Software (Molecular Devices, Sunnyvale, (A, USA). Raw data were processed and analyzed with GeneSpring GX 13 (Agilent Technologies, Milano, Italy). To remove unreliable data, all genes from all samples were filtered for quality to include only probe data fulfilling all of the following criteria in all replicates of at least one out of four experimental conditions: the spot had $<3 \%$ of saturated pixels at $532 \mathrm{~nm}$; the spot was not flagged 'bad', 'not found' or 'absent'; the spot was detectable well above background (signal-to-noise ratios at $532 \mathrm{~nm}$ > 10). Data filtering listed 29892 probes out of a total of 41012 present on the microarray. Raw data are available in gene expression omnibus database with the accession number GSE67788. Genes in our qualityfiltered data set were screened by a one-way ANOVA using Welch's $t$-test, followed by the Benjamin and Hochberg false discovery rate (FDR) procedure as a multiple testing correction and the Tukey's post hoc test. Genes with a corrected $P$-value $<0.05$ were selected as differentially expressed genes. To analyze gene expression changes in the context of known biological pathways, we used MetaCore (Thomson Reuters, Milano, Italy). $P$-values were calculated using a basic formula for the hypergeometric distribution, where the $P$-value essentially represents the probability of a particular pathway arising by chance. To limit the possible number of type I errors among the results, an FDR threshold of 0.05 was used to identify statistically significant pathways. To unravel the genetic networks involved, reduce redundancy of deregulated pathways and simplify their comprehension, the most significant variations were summarized in hand-curated figures drawn by Pathway Map Creator (Thomson Reuters, Milano, Italy). Statistically significant genes and pathways are listed in Supplementary Data S9.

Quantitative real-time reverse transcription-PCR. Although our data represent the average gene expression from four replicates, we confirmed the reliability of the microarray data by performing quantitative real-time RT-PCR according to the protocol described previously ${ }^{1}$ and using the primers listed in Supplementary Table 1.

\section{ACKNOWLEDGEMENTS}

We gratefully acknowledge Maria Patrizia D'Angelo, Alfia Corsino, Cristina Calì and Francesco Marino for their administrative and technical support.

\section{COMPETING INTERESTS}

The authors declare no conflict of interest. 


\section{REFERENCES}

1 Maino B, D'Agata V, Severini C, Ciotti MT, Calissano P, Copani A et al. Igf1 and Pacap rescue cerebellar granule neurons from apoptosis via a common transcriptional program. Cell Death Discov 2015; 1: 15029.

2 D'Mello SR, Galli C, Ciotti T, Calissano P. Induction of apoptosis in cerebellar granule neurons by low potassium: inhibition of death by insulin-like growth factor I and CAMP. Proc Natl Acad Sci USA 1993; 90: 10989-10993.

3 Cavallaro S, Copani A, D'Agata V, Musco S, Petralia S, Ventra C et al. Pituitary adenylate cyclase activating polypeptide prevents apoptosis in cultured cerebellar granule neurons. Mol Pharmacol 1996; 50: 60-66.

4 Canonico PL, Copani A, D'Agata V, Musco S, Petralia S, Travali S et al. Activation of pituitary adenylate cyclase-activating polypeptide receptors prevents apoptotic cell death in cultured cerebellar granule cells. Ann NY Acad Sci 1996; 805: 470-472.

5 Maino B, Ciotti MT, Calissano P, Cavallaro S. Transcriptional analysis of apoptotic cerebellar granule neurons following rescue by gastric inhibitory polypeptide. Int J Mol Sci 2014; 15: 5596-5622.

6 Pieri M, Amadoro G, Carunchio I, Ciotti MT, Quaresima S, Florenzano F et al. SP protects cerebellar granule cells against beta-amyloid-induced apoptosis by down-regulation and reduced activity of Kv4 potassium channels. Neuropharmacology 2010; 58: 268-276.

7 Cavallaro S. Cracking the code of neuronal apoptosis and survival. Cell Death Dis 2015; 6: e1963.

8 Ciranna L, Cavallaro S. Opposing effects by pituitary adenylate cyclase-activating polypeptide and vasoactive intestinal peptide on hippocampal synaptic transmission. Exp Neurol 2003; 184: 778-784.

9 Cavallaro S. Genomic analysis of transcriptional changes underlying neuronal apoptosis. Methods Mol Biol 2015; 1254: 141-151.

10 Cavallaro S, D'Agata V, Alessi E, Coffa S, Alkon DL, Manickam P et al. Gene expression profiles of apoptotic neurons. Genomics 2004; 84: 485-496.

11 Paratore S, Parenti R, Torrisi A, Copani A, Cicirata F, Cavallaro S. Genomic profiling of cortical neurons following exposure to beta-amyloid. Genomics 2006; 88: 468-479.

12 Severini C, Improta G, Falconieri-Erspamer G, Salvadori S, Erspamer V. The tachykinin peptide family. Pharmacol Rev 2002; 54: 285-322.

13 Amadoro G, Pieri M, Ciotti MT, Carunchio I, Canu N, Calissano P et al. Substance P provides neuroprotection in cerebellar granule cells through Akt and MAPK/Erk activation: evidence for the involvement of the delayed rectifier potassium current. Neuropharmacology 2007; 52: 1366-1377.

14 Martin B, Lopez de Maturana R, Brenneman R, Walent T, Mattson MP, Maudsley S. Class II G protein-coupled receptors and their ligands in neuronal function and protection. Neuromol Med 2005; 7: 3-36.

15 Luo J, Elwood F, Britschgi M, Villeda S, Zhang H, Ding Z et al. Colony-stimulating factor 1 receptor (CSF1R) signaling in injured neurons facilitates protection and survival. J Exp Med 2013; 210: 157-172.

16 Peng YP, Qiu YH, Lu JH, Wang JJ. Interleukin-6 protects cultured cerebellar granule neurons against glutamate-induced neurotoxicity. Neurosci Lett 2005; 374: 192-196.

17 Salonen V, Kallinen S, Lopez-Picon FR, Korpi ER, Holopainen IE, Uusi-Oukari M. AMPA/kainate receptor-mediated up-regulation of GABAA receptor delta subunit mRNA expression in cultured rat cerebellar granule cells is dependent on NMDA receptor activation. Brain Res 2006; 1087: 33-40.

18 Jiang X, Zhu D, Okagaki P, Lipsky R, Wu X, Banaudha K et al. N-methyl-D-aspartate and TrkB receptor activation in cerebellar granule cells: an in vitro model of preconditioning to stimulate intrinsic survival pathways in neurons. Ann NY Acad Sci 2003; 993: 134-145; discussion 59-60.

19 Perez DM, Doze VA. Cardiac and neuroprotection regulated by alpha(1)adrenergic receptor subtypes. J Recept Sig Transd Res 2011; 31: 98-110.

20 Bozzi Y, Borrelli E. Dopamine in neurotoxicity and neuroprotection: what do D2 receptors have to do with it? Trends Neurosci 2006; 29: 167-174.

21 Chen JF, Sonsalla PK, Pedata F, Melani A, Domenici MR, Popoli P et al. Adenosine $\mathrm{A} 2 \mathrm{~A}$ receptors and brain injury: broad spectrum of neuroprotection, multifaceted actions and 'fine tuning' modulation. Progr Neurobiol 2007; 83: 310-331.

22 Gomes E, Papa L, Hao T, Rockwell P. The VEGFR2 and PKA pathways converge at MEK/ERK1/2 to promote survival in serum deprived neuronal cells. Mol Cell Biochem 2007; 305: 179-190.

23 Chong ZZ, Maiese K. Targeting WNT, protein kinase B, and mitochondrial membrane integrity to foster cellular survival in the nervous system. Histol Histopathol 2004; 19: 495-504.

24 Manickam M, Tulsawani R. Survival response of hippocampal neurons under low oxygen conditions induced by Hippophae rhamnoides is associated with JAK/STAT signaling. PLoS One 2014; 9: e87694.

25 Weinreb O, Amit T, Bar-Am O, Chillag-Talmor O, Youdim MB. Novel neuroprotective mechanism of action of rasagiline is associated with its propargyl moiety: interaction of Bcl-2 family members with PKC pathway. Ann NY Acad Sci 2005; 1053: 348-355.

26 Zhu D, Jiang X, Wu X, Tian F, Mearow K, Lipsky RH et al. Inhibition of protein kinase $\mathrm{C}$ promotes neuronal survival in low potassium through an Akt-dependent pathway. Neurotox Res 2004; 6: 281-289.

27 Zundorf G, Reiser G. Calcium dysregulation and homeostasis of neura calcium in the molecular mechanisms of neurodegenerative diseases provide multiple targets for neuroprotection. Antioxidants Redox Signal 2011; 14: $1275-1288$.

28 Yu SP, Canzoniero LM, Choi DW. Ion homeostasis and apoptosis. Curr Opin Cell Biol 2001; 13: 405-411.

29 Strappazzon F, Torch S, Trioulier Y, Blot B, Sadoul R, Verna JM. Survival response-linked Pyk2 activation during potassium depletion-induced apoptosis of cerebellar granule neurons. Mol Cell Neurosci 2007; 34: 355-365.

$30 \mathrm{Wu}$ WB, Huang TF. Activation of MMP-2, cleavage of matrix proteins, and adherens junctions during a snake venom metalloproteinase-induced endothelial cell apoptosis. Exp Cell Res 2003; 288: 143-157.

31 Dere E, Zlomuzica A. The role of gap junctions in the brain in health and disease. Neurosci Biobehav Rev 2012; 36: 206-217.

32 Ashutosh, Chao C, Borgmann K, Brew K, Ghorpade A. Tissue inhibitor of metalloproteinases-1 protects human neurons from staurosporine and HIV-1induced apoptosis: mechanisms and relevance to HIV-1-associated dementia. Cell Death Dis 2012; 3: e332.

33 Hirokawa N, Niwa S, Tanaka Y. Molecular motors in neurons: transport mechanisms and roles in brain function, development, and disease. Neuron 2010; 68: 610-638.

34 Schmieg N, Menendez G, Schiavo G, Terenzio M. Signalling endosomes in axonal transport: travel updates on the molecular highway. Semin Cell Dev Biol 2013; 27 32-43.

35 Li J, Liu N, Wang Y, Wang R, Guo D, Zhang C. Inhibition of EphA4 signaling after ischemia-reperfusion reduces apoptosis of CA1 pyramidal neurons. Neurosci Lett 2012; 518: 92-95.

36 Li Y, Xu C, Schubert D. The up-regulation of endosomal-lysosomal components in amyloid beta-resistant cells. J Neurochem 1999; 73: 1477-1482.

37 Bhattacharya S, Stewart BA, Niemeyer BA, Burgess RW, McCabe BD, Lin P et al. Members of the synaptobrevin/vesicle-associated membrane protein (VAMP) family in Drosophila are functionally interchangeable in vivo for neurotransmitter release and cell viability. Proc Natl Acad Sci USA 2002; 99 13867-13872.

38 Stelmashook EV, Lozier ER, Goryacheva ES, Mergenthaler P, Novikova SV, Zorov DB et al. Glutamine-mediated protection from neuronal cell death depends on mitochondrial activity. Neurosci Lett 2010; 482: 151-155.

39 Tabuchi A, Funaji K, Nakatsubo J, Fukuchi M, Tsuchiya T, Tsuda M. Inactivation of aconitase during the apoptosis of mouse cerebellar granule neurons induced by a deprivation of membrane depolarization. J Neurosci Res 2003; 71: 504-515.

40 Atlante A, de Bari L, Bobba A, Marra E, Calissano P, Passarella S. Cytochrome C released from cerebellar granule cells undergoing apoptosis or excytotoxic death, can generate protonmotive force and drive ATP synthesis in isolated mitochondria. J Neurochem 2003; 86: 591-604.

41 Weisova P, Davila D, Tuffy LP, Ward MW, Concannon CG, Prehn JH. Role of $5^{\prime}$-adenosine monophosphate-activated protein kinase in cell survival and death responses in neurons. Antioxidants Redox Signal 2011; 14 1863-1876.

42 Piccioli P, Porcile C, Stanzione S, Bisaglia M, Bajetto A, Bonavia R et al. Inhibition of nuclear factor-kappaB activation induces apoptosis in cerebellar granule cells. J Neurosci Res 2001; 66: 1064-1073.

43 Tain LS, Mortiboys H, Tao RN, Ziviani E, Bandmann O, Whitworth AJ. Rapamycin activation of 4E-BP prevents parkinsonian dopaminergic neuron loss. Nat NeurosCi 2009; 12: 1129-1135.

44 Boutillier AL, Trinh E, Loeffler JP. Selective E2F-dependent gene transcription is controlled by histone deacetylase activity during neuronal apoptosis. J Neurochem 2003; 84: 814-828.

45 Mattson MP. Methylation and acetylation in nervous system development and neurodegenerative disorders. Ageing Res Rev 2003; 2: 329-342.

46 Martin-Romero FJ, Santiago-Josefat B, Correa-Bordes J, Gutierrez-Merino C, Fernandez-Salguero P. Potassium-induced apoptosis in rat cerebellar granule cells involves cell-cycle blockade at the G1/S transition. J Mol Neurosci 2000; 15: $155-165$.

47 O'Hare MJ, Hou ST, Morris EJ, Cregan SP, Xu Q, Slack RS et al. Induction and modulation of cerebellar granule neuron death by E2F-1. J Biol Chem 2000; 275: 25358-25364.

48 Akashiba H, Matsuki N, Nishiyama N. P27 small interfering RNA induces cell death through elevating cell cycle activity in cultured cortical neurons: a proof-ofconcept study. Cell Mol Life Sci 2006; 63: 2397-2404. 
49 Martin-Romero FJ, Garcia-Martin E, Gutierrez-Merino C. Inhibition of oxidative stress produced by plasma membrane NADH oxidase delays low-potassiuminduced apoptosis of cerebellar granule cells. J Neurochem 2002; 82: 705-715.

50 Portt L, Norman G, Clapp C, Greenwood M, Greenwood MT. Anti-apoptosis and cell survival: a review. Biochim Biophys Acta 2011; 1813: 238-259.

51 Neutzner A, Li S, Xu S, Karbowski M. The ubiquitin/proteasome system-dependent control of mitochondrial steps in apoptosis. Semin Cell Dev Biol 2012; 23: 499-508.

52 D'Agata V, Grimaldi M, Pascale A, Cavallaro S. Regional and cellular expression of the parkin gene in the rat cerebral cortex. Eur J Neurosci 2000; 12: 3583-3588.
This work is licensed under a Creative Commons Attribution 4.0 International License. The images or other third party material in this article are included in the article's Creative Commons license, unless indicated otherwise in the credit line; if the material is not included under the Creative Commons license, users will need to obtain permission from the license holder to reproduce the material. To view a copy of this license, visit http://creativecommons.org/licenses/ by/4.0/

(c) The Author(s) 2016

Supplementary Information accompanies the paper on the Cell Death and Discovery website (http://www.nature.com/cddiscovery) 